PROCEEDINGS OF THE

AMERICAN MATHEMATICAL SOCIETY

Volume 129, Number 6, Pages 1689-1696

S 0002-9939(00)05744-0

Article electronically published on November 2, 2000

\title{
RESIDUALLY FINITE DIMENSIONAL AND AF-EMBEDDABLE $C^{*}$-ALGEBRAS
}

\author{
HUAXIN LIN
}

(Communicated by David R. Larson)

\begin{abstract}
We show that every separable nuclear residually finite dimensional $C^{*}$-algebras satisfying the Universal Coefficient Theorem can be embedded into a unital separable simple AF-algebra.
\end{abstract}

The problem of determining when a $C^{*}$-algebra can be embedded as a $C^{*}$ subalgebra of an AF-algebra has been studied for a while (for example, in $\mathrm{Pi}$, $[\mathrm{Vo},[\mathrm{Sp}],[\mathrm{Br}]$ and $[\mathrm{D}]$ - to name a few). J. Spielberg showed in $[\mathrm{Sp}]$, among other related things, that every separable CCR $C^{*}$-algebra can be embedded into an AFalgebra. In [D], it is shown that a separable nuclear residually finite dimensional $C^{*}$-algebra can be embedded into an AF-algebra if it is homotopically dominated by an AF-algebra. Consequently, from a result of Spielberg, Dadarlat shows (in (D) that the AF-embeddability of a residually finite dimensional $C^{*}$-algebra $A$ (say in the "bootstrap" class of [RS] depends only on the homotopy type of $A$. The renewed interest in residually finite dimensional $C^{*}$-algebras arises from the result of Blackadar and Kirchberg (BBK1] and [BK2]) that simple separable nuclear quasidiagonal $C^{*}$-algebras are inductive limits of nuclear RFD $C^{*}$-algebras.

Since a $C^{*}$-subalgebra of a nuclear $C^{*}$-algebra must be exact ([K1]), non-exact $C^{*}$-algebras cannot be embedded into $\mathrm{AF}$-algebras. So, we will consider nuclear $C^{*}$-algebras only. In this short note, we use recent results $([\operatorname{Ln} 3 \mid)$ in classification of nuclear $C^{*}$-algebras to show that, in fact, every separable nuclear residually finite dimensional $C^{*}$-algebra can be embedded into a simple AF-algebra if it satisfies the Universal Coefficient Theorem (for example, if it is in the so-called "bootstrap" class of $C^{*}$-algebras).

Definition 1. Let $A$ be a $C^{*}$-algebra. An irreducible representation of $A, \pi$, is said to be finite dimensional, if $\pi(A)$ is finite dimensional. A $C^{*}$-algebra $A$ is said be residually finite dimensional (RFD) if there is a separating family of finite dimensional irreducible representations of $A$.

A separable RFD $C^{*}$-algebra has a separating sequence of finite dimensional irreducible representations.

Definition 2. Denote by $\mathcal{N}_{1}$ the bootstrap class of $C^{*}$-algebras (see [RS]). Denote by $\mathcal{N}$ the class of $C^{*}$-algebras for which the Universal Coefficient Theorem (UCT)

Received by the editors July 22, 1998 and, in revised form, September 13, 1999.

2000 Mathematics Subject Classification. Primary 46L05, 46L35.

Key words and phrases. AF-embedding, TAF $C^{*}$-algebra.

Research partially supported by NSF grants DMS 9801482.

(C)2000 American Mathematical Society 
holds. Every $C^{*}$-algebra in $\mathcal{N}_{1}$ satisfies the UCT ([RS] $)$. In fact, $A \in \mathcal{N}$ if $A$ is $k k$ equivalent to a $C^{*}$-algebra in $\mathcal{N}_{1} \cdot \mathcal{N}_{1}$ contains all separable type I $C^{*}$-algebras, and is closed under inductive limits, hereditary subalgebras, quotients, tensor products, cross products with $\mathbf{Z}$, as well as extensions.

3. Let $C \in \mathcal{N}$ be a unital separable residually finite dimensional $C^{*}$-algebra. Let $s(C)$ (with the weak-topology) be the set of tracial states defined by $\operatorname{tr} \circ \pi$, where $\pi$ is an irreducible representation of finite rank and $t r$ is the standard tracial state on $\pi(C)$. There is a positive homomorphism $\delta_{C}: K_{0}(C) \rightarrow C(s(A))$ defined by $f \mapsto f(t)(t \in s(A))$.

Let $A$ be a unital separable stably finite $C^{*}$-algebra and $T(A)$ be its tracial state space. Let $\operatorname{Aff}(T(A))$ be the continuous affine functions on $T(A)$. Define $\rho_{A}: K_{0}(A) \rightarrow A f f(T(A))$ by $f \mapsto f(t)(t \in T(A))$.

Definition 4 (2.1 in [Ln2]). Let $A$ be a unital simple $C^{*}$-algebra. We say that $A$ is tracially approximately finite dimensional (TAF for brevity) if it satisfies the following: For any $\varepsilon>0$ and any finite subset $\mathcal{F}$ of $A$ which contains a non-zero element $x_{1}$ and a nonzero positive element $a \in A_{+}$there exists a finite dimensional $C^{*}$-subalgebra $B \subset A$ with $p=1_{B}$ such that

(1) $\|p x-x p\|<\varepsilon$ for all $x \in \mathcal{F}$;

(2) $p x p \subset_{\varepsilon} F$ for all $x \in \mathcal{F}$ and $\left\|p x_{1} p\right\| \geq\left\|x_{1}\right\|-\varepsilon$;

(3) $1-p$ is equivalent to a projection in $\overline{a A a}$.

Simple TAF $C^{*}$-algebras are quasidiagonal, have real rank zero, stable rank one, and weakly unperforated $K_{0}$.

5. Let $C \in \mathcal{N}$ be a unital separable residually finite dimensional $C^{*}$-algebra. Fix a sequence of finite dimensional irreducible representations $\left\{\pi_{k}\right\}$ such that $\left\{\pi_{k}\right\}_{k=m}^{\infty}$ is separating for $C$ for all $m$. We also assume that $\left\{t r \circ \pi_{k}\right\}_{k=m}^{\infty}$ is dense in $s(A)$. Suppose that $\pi_{k}$ has rank $r(k)$. In what follows, we may not distinguish $\pi_{k}$ from $\pi_{k} \otimes \operatorname{id}_{K}\left(\right.$ on $\left.C \otimes M_{K}\right)$.

Let $\bar{r}(2)=r(1)+1, \bar{r}(n+1)=(n) !(\bar{r}(n) r(n)(n+1)), n=1,2, \ldots$ Set $R(i)=$ $\bar{r}(i) r(i), i=1,2, \ldots$ Let $C_{1}=C, C_{2}=C_{1} \otimes M_{\bar{r}(2)}, \ldots, C_{n+1}=C_{n} \otimes M_{\bar{r}(n+1)}, \ldots$ Define $j_{n, n+1}: C_{n} \rightarrow C_{n+1}$ by

$$
f \mapsto \operatorname{diag}\left(f, f, \ldots, f, \pi_{n} \otimes \operatorname{id}_{\bar{r}(n)}(f), \ldots, \pi_{n} \otimes \operatorname{id}_{\bar{r}(n)}(f)\right),
$$

where $f \in C_{n}$ repeats $n$ ! times and $\phi_{n} \otimes \operatorname{id}_{\bar{r}(n)}(f)$ repeats $n(n)$ ! times. Also, the image of $\pi_{n} \otimes \operatorname{id}_{\bar{r}(n)}$ is in $\left(\mathbb{C} \cdot \operatorname{id}_{C_{n}}\right) \otimes M_{\bar{R}(n)}$. Define $A=\lim _{n}\left(C_{n}, j_{n, n+1}\right)$. In what follows, the map $C_{n} \rightarrow A$ will be denoted by $j$.

Lemma 6. $A$ is a unital separable nuclear simple TAF $C^{*}$-algebra with divisible $K_{0}(A)$ and a unique tracial state and which satisfies the UCT.

Proof. Since $A$ is a direct limit of $C_{n}$ and each $C_{n}$ is nuclear and satisfies the UCT, $A$ is separable, nuclear and satisfies the UCT. It follows from 4.2, 4.3 and 4.4 in [n2] that $A$ is a unital simple TAF $C^{*}$-algebra with a unique tracial state. We need only to show that $K_{0}(A)$ is divisible. It suffices to show that, for any projection $p \in M_{K}(A)$ ( $K$ is any positive integer) and any integer $k>0$, there exists a projection $q \in M_{K}(A)$ such that $k[q]=[p]$ in $K_{0}(A)$. Replacing $p$ by an equivalent projection, without loss of generality, we may assume that $p \in j\left(M_{K}\left(C_{n}\right)\right)$ for some large $n$. There exists $m$ such that $k \mid(n+m)$. Let $p^{\prime} \in M_{K}\left(C_{n}\right)$ such that $j\left(p^{\prime}\right)=p$. From the construction in 5 , it is clear that there exists $q^{\prime} \in M_{K}\left(C_{n+m}\right)$ such that $k\left[q^{\prime}\right]=\left[j_{n, n+m}\left(p^{\prime}\right)\right]$ in $K_{0}\left(C_{n+m}\right)$. This implies that $k\left[j\left(q^{\prime}\right)\right]=[p]$. 
7. Fix $m$ and $n>0$. Let $j_{m, m+n}=j_{m+n-1, m+n} \circ \cdots \circ j_{m, m+1}$. Note $C_{m+n}=$ $C_{m} \otimes M_{K(n)}$, where $K(n)=\prod_{i=m}^{m+n-1}(R(i) i !(i+1))$. Thus

$$
j_{m, m+n}(f)=\operatorname{diag}\left(f, f, \ldots, f, \Pi_{m}(f), \Pi_{m+1}(f), \ldots, \Pi_{m+n-1}(f)\right),
$$

where $f$ repeats $\prod_{i=1}^{m+n}(m+i-1)$ many times, $\Pi_{m}$ is

$$
m(m !) \prod_{j=1}^{n-1}(R(m+j)(m+j) !(m+j+1))
$$

copies of $\pi_{m} \otimes \operatorname{id}_{R(m)}, \Pi_{m+1}$ is

$$
(m+1)(m+1) ! \prod_{j=2}^{n-1}(R(m+j)(m+j) !(m+j+1))
$$

copies of $\pi_{m+1} \otimes \operatorname{id}_{R(m)}, \ldots, \Pi_{m+n-1}$ is $(m+n-1)(m+n-1)$ ! copies of $\pi_{m+n-1} \otimes$ $\operatorname{id}_{R(m)}$. Set $A=\lim _{n}\left(C_{n}, j_{n, n+1}\right)$.

Define

$$
\begin{aligned}
a_{1}^{(n)} & =\left(m(m) ! \prod_{j=1}^{n-1}(R(m+j)(m+j) !(m+j+1)) / K(n)=m /(R(m)(m+1),\right. \\
a_{2}^{(n)} & =(m+1)(m+1) ! \prod_{j=2}^{n}(R(m+j)(m+j) !(m+j+1)) / K(n) \\
& =1 / R(m) m !(R(m+1)((m+1)+1)), \ldots
\end{aligned}
$$

and

$$
\begin{aligned}
a_{k}^{(n)}=( & m+k)(m+k) ! /[R(m+k)(m+k) !(m+k+1) \\
& \left.\times \prod_{j=1}^{k-1}(R(m+j)(m+j)+1)\right] \\
=1 /[ & R(m+k-1)(m+k-1) ! R(m+k)(m+k+1) \\
& \left.\times \prod_{j=1}^{k-2} R(m+j)(m+j) !(m+j+1)\right], \ldots
\end{aligned}
$$

Fix $j$, then $a_{j}^{(n)}=a_{j}^{(m+1)}$ for all $n>m$. Thus we set $a_{j}=a_{j}^{(n)}$. Note that $a_{j}^{(n)} \in \mathbb{Q}$. Let $p_{1}, p_{2}, \ldots, p_{r} \in C_{m} \otimes M_{K}$ be nonzero projections and $t r$ be the normalized trace on $\pi_{j} \otimes \operatorname{id}_{\bar{R}(m)}\left(C_{m}\right)(j=m, m+1, \ldots, m+n)$. Set $x_{i j}=\operatorname{tr}\left(\pi_{m+j} \otimes \operatorname{id}_{K}\left(p_{i}\right)\right)$, $i=1,2, \ldots, r$ and $j=1,2, \ldots, n$. So $x_{i j} \in \mathbb{Q}$. Let $P_{m, m+n}: C_{m} \rightarrow C_{m+n}$ be defined by

$$
f \mapsto\left(0, \Pi_{m}(f), \Pi_{m+1}(f), \ldots, \Pi_{m+n}(f)\right)
$$

We compute that

$$
b_{j}^{(n)}=\operatorname{tr}\left(P_{m, m+n} \otimes \operatorname{id}_{K}\left(p_{i}\right)\right)=\sum_{i=1}^{n} x_{i j} a_{j} .
$$


It is important to note that $x_{i j}, a_{j}^{(n)}, b_{j}^{(n)} \in \mathbb{Q}$. Let $\tau$ be the unique tracial state of $A$. Set $\tau\left(j\left(p_{j}\right)\right)=z_{j}$. Note that

$$
\left(\prod_{j=m}^{m+n}(m+j-1)\right) / K(n) \rightarrow 0 \quad(\text { as } n \rightarrow \infty) .
$$

Therefore, it is easy to see that $b_{j}^{(n)} \rightarrow z_{j}$ as $n \rightarrow \infty$.

Lemma 8. Let $\tau$ be the unique tracial state on $A$. Then $\left.\tau\right|_{j\left(C_{m}\right)}$ is in the weak closure of the convex hull of $s\left(C_{m}\right)$. Consequently, $\left.\operatorname{ker} \tau\right|_{j\left(C_{m}\right)} \supset \operatorname{ker} \delta_{C_{m}}$.

Proof. First, if $t \in s\left(C_{m+n}\right)$, it is easy to compute that $\left.t\right|_{j_{m, m+n}\left(C_{m}\right)}$ is in the convex hull of $s\left(C_{m}\right)$. Take a sequence of $\left\{t_{n}\right\} \subset s\left(C_{m+n}\right)$. Extend $t_{n}$ to a state on $A$ and let $s$ be a weak limit of $t_{n}$ (on $A$ ). Then $s$ is a tracial state on $A$. Therefore $s=\tau$. This implies that $\left.\tau\right|_{j\left(C_{m}\right)}$ is in the weak closure of the convex hull of $s\left(C_{m}\right)$.

Lemma 9. For any finitely generated subgroup $G \subset K_{0}(A)$, there is a finitely generated free subgroup $G_{0} \subset K_{0}\left(C_{m}\right)$ for some $m>0$ which has $\mathbb{Z}$-rank $r$ and is generated by $r$ positive $\mathbb{Z}$-linearly independent elements $f_{1}, \ldots, f_{r} \in G_{0}$ such that $\rho_{A} \circ j_{*}\left(G_{0}\right) \supset \rho_{A}(G)$ and $\rho_{A} \circ j_{*}$ is injective on $G_{0}$.

Proof. Note that $\rho_{A}\left(K_{0}(A)\right)$ is a divisible subgroup of $\mathbb{R}$. Let $g_{1}, g_{2}, \ldots, g_{r}$ be $\mathbb{Q}$ linearly independent elements in $\rho_{A}\left(K_{0}(A)\right)_{+}$such that $\bigoplus_{i=1}^{r} \mathbb{Z} g_{i} \supset \rho_{A}(G)$. Let $\imath: \oplus_{i=1}^{r} \mathbb{Z} g_{i} \rightarrow K_{0}(A)$ be an injection such that $\rho_{A} \circ \imath=\operatorname{id}_{\oplus_{i=1}^{r}} \mathbb{Z} g_{i}$. Note that $\imath\left(g_{i}\right)$ is positive $(i=1,2, \ldots, r)$. Therefore there are $f_{1}, f_{2}, \ldots, f_{r} \in K_{0}\left(C_{m}\right)_{+}$for some large $m$ such that $j_{*}\left(f_{i}\right)=\imath\left(g_{i}\right), i=1,2, \ldots, r$. Let $G_{0}$ be the subgroup generated by $f_{1}, \ldots, f_{r}$. We see that $G_{0}$ has rank $r$ and $f_{1}, f_{2}, \ldots, f_{r} \in K_{0}\left(C_{m}\right)_{+}$are $\mathbb{Z}$-linearly independent.

Lemma 10. Let $F$ be a divisible ordered subgroup of $\mathbb{R}$. Let $\left\{x_{i j}\right\}_{0<i \leq r, 0<j<\infty}$ be an $r \times \infty$ matrix having rank $r$ and with each $x_{i j} \in \mathbb{Q}_{+}$, and let $\left\{a_{j}^{(n)}\right\}$ be sequences of positive rational numbers such that $a_{j}^{(n)} \rightarrow a_{j}(>0)$ as $n \rightarrow \infty$. For each $n$,

$$
\left(x_{i j}\right)_{r \times n} v_{n}=y_{n},
$$

where $v_{n}=\left(a_{j}^{(n)}\right)_{n \times 1}$ is an $n \times 1$ column vector and $y_{n}=\left(b_{i}^{(n)}\right) \in \mathbb{Q}^{r}$ is an $r \times 1$ column vector.

Suppose that $y_{n} \rightarrow z$ for some $z=\left(z_{j}\right)_{r \times 1} \in F^{r}$ with $z_{j} \geq 0$ (in $\mathbb{R}^{r}$ norm). Then for some sufficiently large $n$, there is $u=\left(c_{j}\right)_{n \times 1} \in F_{+}^{n}\left(c_{j}>0\right)$ such that

$$
\left(x_{i j}\right)_{r \times n} u=z .
$$

Proof. To save notation, without loss of generality, we may assume that $\left(x_{i j}\right)_{r \times r}$ has rank $r$. Set $A_{n}=\left(x_{i j}\right)_{r \times n}(n \geq r)$. Then there exists an invertible matrix $B \in$ $M_{r}(\mathbb{Q})$ (which does not depend on $n$ ) such that $B A_{n}=C_{n}$, where $C=\left(c_{i j}\right)_{r \times n}$, $c_{i i}=1$ for $i=1,2, \ldots, r$, and $c_{i j}=0$ if $i \neq j, j=1,2, \ldots, r$, and $c_{i j} \in \mathbb{Q}$. Let $I_{r}$ be the $r \times r$ identity matrix. We may write

$$
C_{n}=\left(I_{r}, D_{n}^{\prime}\right),
$$

where $D_{n}^{\prime}$ is a $r \times(n-r)$ matrix. Thus we have

$$
C_{n} v_{n}=B y_{n} \quad \text { and } \quad I_{r} v_{n}^{\prime}=B y_{n}-D_{n} v_{n},
$$


where $v_{n}^{\prime}=\left(a_{1}^{(n)}, a_{2}^{(n)}, \ldots, a_{r}^{(n)}\right)$ (as a column) and $D_{n}=\left(0, D_{n}^{\prime}\right)$ is a $r \times n$ matrix. Note that for any $n \times 1$ column vector $v$ with the form $\left(t_{1}, t_{2}, \ldots, t_{r}, a_{r+1}^{(n)}, a_{r+2}^{(n)}, \ldots\right.$, $\left.a_{n}^{(n)}\right), D_{n} v=D_{n} v_{n}$. Since $a_{j}^{(n)} \rightarrow a_{j}>0$, there is an $N_{1}>0$ such that

$$
a_{j}^{(n)} \geq a_{j} / 2>0
$$

for all $n \geq N_{1}$ and $j=1,2, \ldots, r$. Let $0<\varepsilon<\min \left\{a_{j} / 4: j=1,2, \ldots, r\right\}$. There is $N_{2}>0$ such that

$$
\left\|B y_{n}-B z\right\|_{\infty}<\varepsilon
$$

if $n \geq N_{2}$. Set $N=\max \left\{N_{1}, N_{2}\right\}$. Let $u^{\prime}=\left(c_{1}, c_{2}, . ., c_{r}\right)$ (column vector) satisfy the equation

$$
I_{r} u^{\prime}=B z-D_{n} v_{n}
$$

Since $I_{r} v_{n}^{\prime}=v_{n}^{\prime}$ and $I_{r} u^{\prime}=u^{\prime}$, we have

$$
\left\|u^{\prime}-v^{\prime}\right\|_{\infty}<\varepsilon
$$

if $n \geq N$. Therefore $c_{j}>0$ for $j=1,2, \ldots, r$. Set $u=\left(c_{1}, \ldots, c_{r}, a_{r+1}^{(n)}, a_{r+2}^{(n)}, \ldots, a_{n}^{(n)}\right)$. Then

$$
I_{r} u^{\prime}=B z-D_{n} u
$$

$(n \geq N)$. Since $B \in M_{r}(\mathbb{Q}), D_{n} u=D_{n} u_{n} \in \mathbb{Q}^{r}, z \in F^{r}$ and $F$ is divisible, we conclude that $u^{\prime} \in F^{r}$. Since $D_{n}=C_{n}-I_{r}$, we have

$$
C_{n} u=B z \text {. }
$$

Finally, since $B$ is invertible, we have

$$
A_{n} u=z .
$$

11. Let $B$ be a unital separable simple AF-algebra with $\left(K_{0}(B), K_{0}(B)_{+},\left[1_{B}\right]\right)=$ $\left(\rho_{A}\left(K_{0}(A)\right), \rho_{A}\left(K_{0}(A)\right)_{+}, 1\right)$. Let $\alpha_{0}: K_{0}(A) \rightarrow K_{0}(B)$ be the positive homomorphism defined by $\rho_{A}$. By the Universal Coefficient Theorem, there is $\alpha \in$ $K L(A, B)_{+}$such that $\left.\alpha\right|_{K_{0}(A)}=\alpha_{0}$. As in [DL], we identify $K L(A, B)$ with $\operatorname{Hom}_{\Lambda}(\underline{K}(A), \underline{K}(B))$. An element $\alpha \in K L(A, B)_{+}$is an element such that $\alpha\left(K_{0}(A) \backslash\{0\}\right) \subset\left(K_{0}(A) \backslash\{0\}\right)$.

Since $K_{1}(B)=0$ and $\operatorname{Tor}\left(K_{0}(B)\right)=0$, from the following commutative diagram,

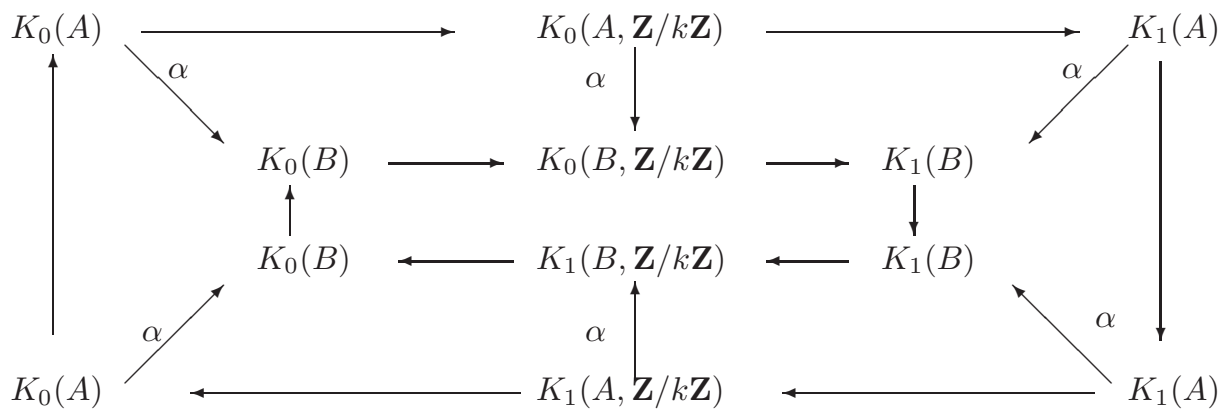


(the map from $K_{i}(-)$ to $K_{i}(-)$ is the multiplication by $k$ ), we have

$$
\left.\alpha\right|_{K_{1}(A)}=0 \text { and }\left.\quad \alpha\right|_{K_{1}((A, \mathbb{Z} / k \mathbb{Z})}=0 .
$$

We claim that

$$
\left.\alpha\right|_{K_{0}(A, \mathbb{Z} / k \mathbb{Z})}=0
$$

for all $k>0$.

Since $\rho_{A}\left(K_{0}(A)\right)=K_{0}(B)$ is divisible, $K_{0}(B) / k K_{0}(B)=0$. Then $\left(\right.$ since $K_{1}(B)$ $=0)$

$$
K_{0}(B, \mathbb{Z} / k \mathbb{Z})=0
$$

Therefore $\left.\alpha\right|_{K_{0}(A, \mathbb{Z} / k \mathbb{Z})}=0$.

In [Ln3], we show the following.

Theorem 12. Let $A$ and $B$ be unital separable simple nuclear TAF $C^{*}$-algebras satisfying the UCT. Let $\alpha \in K L(A, B)_{+}$. Suppose that for every finite subset $\mathcal{G} \subset$ $K_{i}(A, \mathbb{Z} / k \mathbb{Z})(i=0,1, k=0,1, \ldots)$, there exists a sequence of contractive completely positive linear maps $L_{n}: A \rightarrow B$ such that

$$
\left\|L_{n}(a b)-L_{n}(a) L_{n}(b)\right\| \rightarrow 0 \quad(\text { as } n \rightarrow \infty)
$$

and

$$
\left.\left[L_{n}\right]\right|_{G}=\left.\alpha\right|_{G}
$$

Then there is a homomorphism $h: A \rightarrow B$ such that $[h]=\alpha$. (see 1.8 in [Ln3] for the definition of $\left.\left.\left[L_{n}\right]\right|_{G}\right)$.

13. We need only a version of Theorem 12 in which $B$ is an AF-algebra. In the next theorem, we let $B$ be a unital separable simple AF-algebra with

$$
\left(K_{0}(B), K_{0}(B)_{+},\left[1_{B}\right]\right)=\left(\rho_{A}(A), \rho_{A}(A)_{+}, 1\right) .
$$

Let $\alpha \in K L(A, B)_{+}$be as in 11, From[11, we only need to consider $G \subset K_{0}(A)$.

Theorem 14. Every unital separable residually finite dimensional $C^{*}$-algebra in $\mathcal{N}$ can be embedded into a unital simple AF-algebra.

Proof. From the construction in $5 . C$ maps into $A$. Since each $j_{n, n+1}$ is injective, we see that $C$ is embedded into $A$. Let $B$ be as in 11. It suffices to show that $A$ can be embedded into $B$. Let $\alpha$ be as in 13 . Let $G$ be any finitely generated subgroup of $K_{0}(A)$. By 11, Theorem 12 and 13 , to show that $A$ can be embedded into $B$, it suffices to show that there is a sequence of contractive completely positive linear maps $L_{n}: A \rightarrow B$ such that

$$
\left\|L_{n}(a b)-L_{n}(a) L_{n}(b)\right\| \rightarrow 0
$$

for all $a, b \in A$ and

$$
\left.\left[L_{n}\right]\right|_{G}=\left.\alpha\right|_{G}
$$

Let $m, G_{0}$ and $f_{1}, \ldots, f_{r}$ be as in Lemma 9, There are $z_{1}, z_{2}, \ldots, z_{r} \in \rho_{B}(B)_{+} \backslash\{0\}=$ $\rho_{A}(A)_{+} \backslash\{0\}$ such that

$$
\rho_{A} \circ j_{*}\left(f_{i}\right)=z_{i} \quad(i=1,2, \ldots, r) .
$$

Note that $\left.\left(j_{m, m+l}\right)\right|_{G_{0}}$ is injective. So we may assume that there are projections $p_{1}, p_{2}, \ldots, p_{r} \in C_{m} \otimes M_{K}$ such that $\left[p_{i}\right]=f_{i}$ in $K_{0}\left(C_{m}\right), i=1,2, \ldots, r$. We now use the notation developed in $\left[\right.$. Since $f_{1}, \ldots, f_{r}$ are $\mathbb{Z}$-linearly independent, by Lemma 
$8 \delta_{C_{m}}\left(f_{1}\right), \ldots, \delta_{C_{m}}\left(f_{r}\right)$ are $\mathbb{Z}$-linearly independent. Since $\left\{t r \circ \pi_{n}\right\}$ is dense in $s(C)$, we conclude that $\left(x_{i j}\right)$ has rank $r$. By Lemma10, (with $z=\left(z_{1}, z_{2}, \ldots, z_{r}\right)$, a column vector in $\left.\rho_{A}(A)^{r}\right)$, there is $u \in\left(\rho_{A}(A)^{n}\right)_{+}$such that

$$
\left(x_{i j}\right)_{r \times n} u=z .
$$

Let $D=\bigoplus_{i=m}^{m+n} \pi_{i} \otimes \operatorname{id}\left(C_{m}\right)$. Note $K_{0}(D)=\mathbb{Z}^{n}$. Define a positive map $\lambda: K_{0}(D) \rightarrow$ $\rho_{B}(B)=\rho_{A}(A)$ by

$$
\left(l_{1}, l_{2}, \ldots, l_{n}\right)=\sum_{j=1}^{n}\left(\frac{l_{j}}{R(m+j-1)}\right) c_{j}
$$

$\left(u=\left(c_{1}, c_{2}, \ldots, c_{n}\right) \in\left(\rho_{A}(A)^{n}\right)_{+}\right)$. It is well known that there exists a homomorphism $h: D \rightarrow B$ such that

$$
\left.[h]\right|_{K_{0}(D)}=\lambda .
$$

Let $L_{n}^{\prime}=h \circ \bigoplus_{j=m}^{m+n} \pi_{i}: C_{m} \rightarrow B$. Note for each $i$,

$$
\left(\bigoplus_{j=m}^{m+n} \pi_{j}\right)_{*}\left(f_{i}\right)=\left(R(m) x_{i 1}, R(m+1) x_{i 2}, \ldots, R(m+n) x_{i n}\right)
$$

and

$$
\rho_{B} \circ\left[L_{n}^{\prime}\right]\left(f_{i}\right)=\sum_{j=1}^{n} x_{i j} c_{j}=z_{i}
$$

Thus

$$
\left.\left[L_{n}^{\prime}\right]\right|_{G_{0}}=\left.\alpha \circ(j)_{*}\right|_{G_{0}} .
$$

For any $\varepsilon_{n}>0$ and any finite subset $\mathcal{F} \subset C_{m}$, since $C_{m}$ is nuclear, there exists a contractive completely positive linear map $L_{n}: A \rightarrow B$ (see 3.2 (2) in [Ln3]) such that

$$
\left\|L_{n}(a)-L_{n}^{\prime}(a)\right\|<\varepsilon_{n}
$$

for all $a \in \mathcal{F}$. Thus (by considering the finitely many generators and by choosing sufficiently small $\varepsilon_{n}$ - see 1.8 in [Ln] )

$$
\left.\left[L_{n}\right]\right|_{G}=\left.\alpha\right|_{G} .
$$

Since we can choose any large $m$ to start, one sees that the theorem follows.

Corollary 15 (cf. 7 in $(\mathrm{D}]$ ). Let $A$ be a separable $R A F C^{*}$-algebra. Then the cone over $A, C A$ and the suspension of $A, S A$, are AF-embeddable.

Proof. First, we note that $C A$ is a separable RAF $C^{*}$-algebra. Since $C A=$ $C_{0}(0,1] \otimes A$ is homotopic to $\{0\}, C A \in \mathcal{N}$. So the corollary follows from Theorem 14 


\section{REFERENCES}

[BK1] B. Blackadar and E. Kirchberg Generalized inductive limits of finite-dimensional C* algebras, Math. Ann. 307 (1997), 343-380. MR 98c:46112

[BK2] B. Blackadar and E. Kirchberg, Inner quasidiagonality and strong NF algebras, preprint.

[Br] N. P. Brown, AF embeddability of cross product of AF algebras by the integers, J. Funct. Anal. 160 (1998), 150-175. CMP 99:06

[D] M. Dadarlat, Residually finite dimensional $C^{*}$-algebras, Cont. Math., Amer. Math. Soc., 228 (1998), 45-50. MR 99m:46133

[DE] M. Dadarlat and S. Eilers, On the classification of nuclear $C^{*}$-algebras, preprint 1998.

[DL] M. Dadarlat and T. Loring, A universal multi-coefficient theorem for the Kasparov groups, Duke J. Math. 84 (1996), 355-377. MR 97f:46109

[El11] G. A. Elliott, On the classification of inductive limits of sequences of semi-simple finite dimensional algebras, J. Algebra 38 (1976), 29-44. MR 53:1279

[El12] G. A. Elliott, On the classification of $C^{*}$-algebras of real rank zero, J. Reine Angew. Math. 443 (1993), 179-219. MR 94i:46074

[GH] K. Goodearl and D. Handelman, Rank functions and $K_{0}$ of regular rings, J. Pure Appl. Algebra 7 (1976), 195-216. MR 52:10794

[K1] E. Kirchberg, On non-semisplit extensions, tensor products and exactness of group $C^{*}$ algebras, Invent. Math. 112 (1993), 449-489. MR 94d:46058

[Ln1] H. Lin Classification of simple $C^{*}$-algebras with unique traces, Amer. J. Math. 120 (1998), 1289-1315. CMP 99:04

[Ln2] H. Lin, Tracially AF $C^{*}$-algebras, Trans. Amer. Math. Soc., to appear.

[Ln3] H. Lin, Classification of simple TAF $C^{*}$-algebras, Can. J. Math., to appear.

[Pi] M. Pimsner, Embedding some transformation group $C^{*}$-algebras into AF algebras, Ergod. Th. Dynam. Sys. 3 (1983), 613-626. MR 86d:46054

[RS] J. Rosenberg and C. Schochet, The Kunneth theorem and the universal coefficient theorem for Kasparov's generalized functor, Duke Math. J. 55 (1987), 431-474. MR 88i:46091

[Sp] J. S. Spielberg, Embedding $C^{*}$-algebra extensions into AF-algebras, J. Funct. Anal. 81 (1988), 325-344. MR 90a:46154

[Vo] D. Voiculescu, Almost inductive limit automorphisms and embedding into AF-algebras, Ergod. Th. Dynam. Sys. 6 (1986), 475-484. MR 88k:46073

Department of Mathematics, East China Normal University, Shanghai, China

Current address: Department of Mathematics, University of Oregon, Eugene, Oregon 974031222

E-mail address: hxlin@noether.uoregon.edu 